\title{
Bolstering Cognitive Resilience via Train-the-Trainer Delivery of Mindfulness Training in Applied High-Demand Settings
}

\author{
Amishi P. Jha ${ }^{1} \cdot$ Anthony P. Zanesco ${ }^{1}$ Ekaterina Denkova ${ }^{1}$ (D) - Alexandra B. Morrison ${ }^{2} \cdot$ Nicolas Ramos $^{1}$. \\ Keith Chichester ${ }^{1}$. John W. Gaddy ${ }^{3,4} \cdot$ Scott L. Rogers ${ }^{5}$
}

Published online: 19 December 2019

(C) The Author(s) 2019

\begin{abstract}
Objectives Mindfulness training (MT) guidelines recommend that trainers have familiarity and knowledge of the training group as well as extensive MT expertise. Herein, a "train-the-trainer" (TTT) dissemination model was investigated for military service members whose access to MT is threatened by a scarcity of qualified trainers.

Methods US Army Master Resilience Trainer-Performance Experts (PEs), who had extensive familiarity with soldiers but no prior MT experience, participated in an MT practicum, and then delivered a 4-week MT program (Mindfulness-Based Attention Training, MBAT) contextualized for military personnel. Soldiers $(n=180)$ undergoing intensive military field training over the study interval were recruited as participants. MBAT was delivered to soldiers by PEs $(n=89)$ or by a trainer with extensive MT experience (Mindfulness Expert; ME, $n=45$ ) but no military familiarity. The remaining participants served as no-training controls (NTC, $n=46$ ). Soldiers' performance on sustained attention and working memory (WM) tasks was assessed before (week 0, T1) and after MBAT delivery (week 5, T2), and again 4 weeks later (week 10, T3).

Results For all participants, sustained attention and WM performance declined over the high-demand field training interval $(p<0.001)$. Yet, the PE group declined significantly less in attentional $(p=0.040)$ and WM $(p<0.001)$ performance relative to the other groups.

Conclusions These results suggest that TTT delivery of short-form MT by context-familiar trainers may be an expeditious route by which to increase access to MT in the service of promoting cognitive resilience in high-demand groups.
\end{abstract}

Keywords Performance $\cdot$ High stress $\cdot$ Attention $\cdot$ Working memory $\cdot$ Mind wandering $\cdot$ Military $\cdot$ Cognitive degradation $\cdot$ Cognitive decline

Electronic supplementary material The online version of this article (https://doi.org/10.1007/s12671-019-01284-7) contains supplementary material, which is available to authorized users.

Amishi P. Jha

a.jha@miami.edu

1 Department of Psychology, University of Miami, Coral Gables, FL, USA

2 Department of Psychology, California State University, Sacramento, CA, USA

3 Armed Forces Services Corporation, Fort Stewart, GA, USA

4 Department of Leadership and Integrative Studies, Kennesaw State University, Kennesaw, GA, USA

5 School of Law, University of Miami, Coral Gables, FL, USA
Mindfulness is defined as "a mental mode characterized by attention to present-moment experience without judgment, elaboration, or emotional reactivity" (Jha et al. 2010, p. 54). Mindfulness training (MT) involves a combination of focused attention (FA) and open monitoring (OM) exercises (Lutz et al. 2015), as well as didactic content and discussion on how to cultivate present-centered attention. While distinct, both FA and OM practices are proposed to repeatedly engage and consequently strengthen selective and reflective attentional engagement, maintenance, and monitoring (see Jha et al. 2019, for a detailed description). Since these processes are also necessary for successful performance on cognitive tasks, their repeated engagement during MT exercises is predicted to bolster performance on attention and working memory (WM) tasks (Jha et al. 2019).

Indeed, prior studies of MT investigating its use as a cognitive training tool have demonstrated MT-related benefits on 
attention (e.g., Jha et al. 2015; Zanesco et al. 2013) and WM task performance (e.g., Jha et al. 2010; Jha et al. 2017; Mrazek et al. 2013; but see Morrison et al. 2014). Based on these salutary cognitive effects, there has been growing interest in MT's utility for applied settings.

MT has been offered in many real-world contexts (Good et al. 2015), and one setting where it has shown promise is with active-duty military personnel (e.g., Bijlsma et al. 2019; Jha et al. 2010; Johnson et al. 2014; Meland et al. 2015). The motivation for providing MT to military service members stems, in part, from prior research showing decline in cognitive performance over high-demand intervals, such as survival school training (Morgan et al. 2006), and stress inoculation training (Lieberman et al. 2005). During periods of high pressure, ambiguity, and uncertainty, military service members must rely on selective and sustained attention, as well as WM, to achieve operational goals and make difficult and consequential decisions (Stanley and Jha 2009). These cognitive functions are critical for performance and psychological health as well (e.g., Blacker et al. 2018; Hofmann et al. 2012). As such, their degradation may result in grave performance errors (Gamble et al. 2018; Wilson et al. 2015), as well as increased susceptibility to psychological illness (Marx et al. 2009; Vasterling et al. 1998).

Given MT's potential as a tool for cognitive enhancement (e.g., Zanesco et al. 2018; Jha et al. 2019), it has garnered recent interest as a method by which to bolster cognitive resilience - the ability to maintain or regain cognitive capacities at risk of decline over protracted periods of high demand (Jha et al. 2016). Prior studies examining MT in active-duty service members have shown that it can protect against declines in attention and WM task performance that may occur over high-demand intervals (Jha et al. 2010, 2015, 2016; Jha et al. 2017).

While several of these prior studies have offered MT to military service members in longer formats (e.g., 20-24 h; Jha et al. 2010, 2016; Johnson et al. 2014), protective benefits for attention and WM performance have also been found with short-form MT programs with fewer hours dedicated to inclass sessions (e.g., 8 h; Jha et al. 2015, 2017). Short-form MT is advantageous from a practical implementation standpoint since long-form MT is often prohibitive for broad implementation in many time-pressured settings. Recently, two studies examined changes in cognitive task performance when offering short-form MT to Soldiers over their high-demand predeployment training interval (Jha et al. 2015, 2017). Soldiers received an 8-h, 8-week training-focused vs. didactic-focused MT program or no training at all. The training-focused group demonstrated greater protection from performance decline in sustained attention (Jha et al. 2015) and WM (Jha et al. 2017) compared to the didactic-focused group and the no-training control group.
These results are encouraging regarding the feasibility of offering short-form MT to military populations to protect against cognitive decline over high-demand intervals. Yet, it is important to note that these prior short-form MT programs, as well as other longer-form variants offered to active-duty service members (Jha et al. 2010, 2016; Stanley et al. 2011), were delivered by a single trainer who authored the program that was delivered. This trainer had extensive prior expertise in mindfulness practice, as well as considerable familiarity with military life and training (see Stanley 2014). Such background fully aligns with established trainer guidelines for successful dissemination of MT, which recommend that trainers have extensive expertise in mindfulness-related training competencies as well as knowledge, experience, and professional training related to the specific populations to whom MT will be delivered (Crane et al. 2017).

Nonetheless, individuals with such combined expertise and experience are extremely rare. The paucity of eligible trainers poses a significant challenge for large-scale, rapid dissemination of MT to military groups. Growing interest in broader adoption of MT for military service members (Deuster \& Schoomaker 2015) has prompted interest in determining if individuals who have military context familiarity and experience training service members, but no prior experience with MT or MT delivery, can be trained to effectively deliver short-form MT to military service members. Indeed, there has been recent interest in "trainthe-trainer" (TTT) delivery of mindfulness-informed programs by context-familiar trainers in the civilian (Lyssenko et al. 2016) and military (Zhang et al. 2018) literature. These studies showed that TTT delivery decreased self-reported emotional distress (Lyssenko et al. 2016) and increased self-reported mindfulness (Zhang et al. 2018). Yet, no prior studies have examined if TTT delivery of MT effectively promotes cognitive resilience in active-duty service members.

To investigate this issue, individuals with significant professional experience working with military personnel but no prior mindfulness practice or training competencies were identified and recruited for involvement in the current study. These individuals were Master Resilience TrainerPerformance Experts (MRT-PEs), hereafter referred to as PEs (U.S. Army 2014). Mindfulness-naïve PEs were recruited to participate in an MT practicum, which prepared them to deliver MT to Soldiers. Specifically, they were trained to deliver a short-form 4-week, 8-h training program, referred to as Mindfulness-Based Attention Training (MBAT, see Zanesco et al. 2019).

Recently, MBAT's effectiveness as a cognitive enhancement tool was investigated in Special Operations Forces (SOF), who are selected for service, in part, based on their cognitive ability. The program was delivered by a trainer with extensive MT practice and delivery experience, as well as 
SOF-context familiarity. Indeed, relative to participants in a no-training control group, sustained attention and working memory task performance improved in individuals receiving MBAT delivered over 4 weeks, but not 2 weeks (Zanesco et al. 2019).

The present study aimed to extend and broaden these findings by investigating the feasibility and effectiveness (Bowen et al. 2009) of TTT delivery of MBAT. Two key questions were examined. First, what is the impact of PE-delivered MBAT on attention and WM performance in service members undergoing intensive military field training? Second, does the delivery of MBAT by a mindfulness expert (ME) who lacks military context familiarity result in a similar pattern to that observed in the PE-trained group?

\section{Methods}

The design of the present study comprised two components, which occurred sequentially. First, the MT practicum was delivered to prepare PE and ME trainers to deliver MBAT to soldiers. Second, the MBAT program was delivered by PE and ME trainers to soldiers who were evaluated before and after the training. The methods related to each of these two components are described in detail below.

\section{Methods: MBAT Practicum}

\section{Participants}

Eight PEs $\left(M_{\text {age }}=31.5\right.$ years, $S D=6.37,2$ female) from the US Army's Comprehensive Soldier and Family Fitness (CSF2) program were recruited to participate in the training practicum. As CSF2 instructors, the PEs regularly delivered mental skills training involving resilience and performance enhancement topics to active-duty military service members. This training aims to improve the mental fitness of service members but does not include MT. PEs had several years of experience teaching $(M=6.25$ years, $S D=5.26)$, and specific experience teaching active-duty military personnel $(M=$ 1.61 years, $S D=1.79)$. None of the PE trainers had experience teaching mindfulness-based content at study onset.

In addition, a mindfulness expert (ME) was recruited for involvement in the study based on his prior experience as a mindfulness practitioner and MT program deliverer (age = 59 years, male). The ME was certified to deliver Mindfulness-Based Stress Reduction (MBSR) and had extensive mindfulness delivery experience. He had previously taught 18 MBSR courses (Kabat-Zinn 1990) in health centers and other wellness facilities over 4 years but had no familiarity with military culture or prior experience training active-duty Soldiers.
All participants provided informed consent in accordance with the Institutional Review Board at the University of Miami with oversight from the Human Research Protections Office of the US Department of Defense.

\section{Procedure}

To prepare trainers to deliver the MBAT program, PEs participated in a formal 12-week MBAT training practicum, which consisted of two phases. First, they received a 4-week foundational MT phase, which provided PEs with first-person experience engaging in mindfulness exercises and cultivation of mindfulness skills. Subsequently, PEs, together with the ME, engaged in an 8-week basic teacher training delivery phase, which prepared them to deliver the MBAT program to Soldiers. For more details on the MBAT practicum, see Electronic Supplemental Material.

At the end of the practicum, the practicum instructor confirmed that the PE and ME trainers had all achieved sufficient mastery of the course materials and were well qualified to deliver the training to Soldiers. Of the 8 trained PEs, 4 were not available to deliver training at the collaborating military installation due to geographical constraints. Of the 4 remaining PEs, 2 were assigned as trainers to deliver MBAT to Soldiers because they displayed a strong grasp of the content and evinced a strong personal and professional engagement in the training program. One additional $\mathrm{PE}$ was assigned to assist the ME trainer, and another was assigned to offer logistical support for the testing sessions of the NTC group.

\section{Methods: MBAT Delivery to Soldiers}

\section{Participants}

One hundred eighty healthy active-duty male volunteers from four US Army companies were recruited from a military base in the continental USA. Participants were predominantly infantry Soldiers and were drawn from the same infantry battalion. A small subset $(n=34)$ of participants held support positions with close interactions with the infantry population (e.g., combat medics assigned to an infantry battalion).

Three companies were randomly assigned to receive MBAT from $\mathrm{PE}$ or ME trainers. Soldiers from two companies were assigned to receive MBAT from PE trainers $\left(n=89 ; M_{\text {age }}=\right.$ 23.57 years, $S D=4.16$ ). The PE group comprised two subgroups ( $\mathrm{PE}_{1} n=44$ and $\left.\mathrm{PE}_{2} n=45\right)$, each of which was taught by a different PE trainer. Soldiers from the third company received the MBAT course delivered by the ME trainer $(n=45$; $M_{\text {age }}=23.31$ years, $\left.S D=3.0\right)$. Lastly, Soldiers from a fourth company served as a no-training control (NTC) group ( $n=46$; $M_{\text {age }}=23.48, S D=3.46$ ) and did not participate in any MT but were tested at the assessment sessions. The NTC company was not randomly assigned to this condition as they had prior off-site 
training commitments that precluded participating in MT. Other self-reported demographic variables, including education, ethnicity, military rank, and prior military experience, were well matched between groups (see Table 1).

The group-randomized assignment strategy, which was a requirement set by the collaborating military site, was used so that assessment and training sessions could be scheduled with ease while maintaining organic unit structure. This methodology is consistent with many prior studies (e.g., Adler et al. 2008; Johnson et al. 2014; Jha et al. 2015), and is an acknowledged constraint of working with active-duty military cohorts. Figure 1 depicts the Consolidated Standards of Reporting Trials Flow Diagram (CONSORT; Schulz et al. 2010), indicating the flow of participants across each stage of the study.

All Soldier participants provided informed consent in compliance with the Institutional Review Board of the University of Miami with oversight from the US Department of Defense Human Research Protections Office. All testing and training occurred during participants' duty day. Soldiers were not compensated beyond their wages for participation in the project per Department of Defense regulations regarding Soldier compensation during the duty day.

Table 1 Education, ethnicity, and military experience for study participants

\begin{tabular}{lrrr}
\hline Measure & NTC & \multicolumn{1}{l}{ ME } & \multicolumn{1}{l}{ PE } \\
\hline Education & & & \\
High school diploma/GED & $84.78 \%$ & $80.00 \%$ & $88.76 \%$ \\
Post-secondary or trade & $6.52 \%$ & $8.89 \%$ & $2.25 \%$ \\
$\quad$ school & & & \\
Associate's degree & $4.35 \%$ & $11.11 \%$ & $6.74 \%$ \\
Bachelor's degree & $4.35 \%$ & $0.00 \%$ & $2.25 \%$ \\
Ethnicity & & & \\
Not Hispanic or Latino & $71.74 \%$ & $62.22 \%$ & $70.79 \%$ \\
Hispanic or Latino & $15.22 \%$ & $28.89 \%$ & $19.10 \%$ \\
Unknown or not reported & $13.04 \%$ & $8.89 \%$ & $10.11 \%$ \\
Rank & & & \\
E-1 PVT & $0.00 \%$ & $2.22 \%$ & $1.12 \%$ \\
E-2 PV2 & $10.87 \%$ & $22.22 \%$ & $22.47 \%$ \\
E-3 PFC & $41.30 \%$ & $22.22 \%$ & $24.72 \%$ \\
E-4 CPL & $0.00 \%$ & $0.00 \%$ & $3.37 \%$ \\
E-4 SPC & $39.13 \%$ & $28.89 \%$ & $33.71 \%$ \\
E-5 SGT & $8.70 \%$ & $20.00 \%$ & $10.11 \%$ \\
E-6 SSG & $0.00 \%$ & $4.44 \%$ & $4.49 \%$ \\
Years of service (in years) & $2.76(2.84)$ & $2.51(2.38)$ & $2.52(2.38)$ \\
Combat deployment (in & $0.56(0.81)$ & $0.71(0.87)$ & $0.60(0.91)$ \\
years) & & & \\
\hline
\end{tabular}

Demographic variables are provided for groups (NTC $n=46, \operatorname{ME~} n=45$, and PE $n=89$ ) at group assignment (T1). Percentages are provided for participant education, ethnicity, and military rank. Mean $(S D)$ values are provided for self-reported years of military service and prior combat deployment

\section{Procedure}

Participants received the 4-week, 8-h MBAT program delivered in 2-h training sessions that occurred once a week for 4 consecutive weeks. The training groups were offered identical content and practices and differed only in the expertise of the trainers (PEs vs. ME). Trainers had no prior interaction with participants assigned to their training group and were uninvolved in any data collection procedures. In order to maximize the opportunity for interaction with the trainer, participants attended training classes in cohorts of no more than 15 soldiers.

The MBAT program was developed as a structured and manualized program contextualized for the military environment (see Zanesco et al. 2019, and also Electronic Supplemental Material). MBAT can be classified as a mindfulness-based program (MBP, Crane et al. 2017) because it involves "systematic and sustained training in formal and informal mindfulness meditation practices" (Crane et al. 2017, p 991) as part of its core content. The program emphasizes first-hand mindfulness practice and discussion, and aims to provide Soldiers with cognitive resilience and psychological health enhancement tools for use throughout their careers and in their personal lives.

MBAT comprises four central themes (i.e., concentration, body awareness, open monitoring, and connection). Mindfulness exercises corresponding with these themes were introduced during weekly training sessions (see Electronic Supplemental Material). All participants in the training groups were asked to complete daily mindfulness exercises that corresponded with the weekly course material as part of their "out-of-class" individual mindfulness practice. Participants were provided with an MP3 player containing guided practice recordings and were assigned to practice for $15 \mathrm{~min}$ a day for at least 4 days per week until the end of the study interval.

\section{Measures}

Soldiers were assessed in the week before the training period (week 0, T1), following the training period (week 5, T2) as well as 4 weeks following T2 (week 10, T3). Participants were administered a battery of computerized cognitive tasks (see Fig. 2 for task schematics) and self-report questionnaire assessments. The computerized cognitive tasks were the two primary outcome measures for the present study. An identical assessment battery was utilized at all assessments, with the addition of training and practice feedback questions at T2. Testing was proctored by a team of 2 to 3 experimenters in a group setting of up to 18 participants. Each session lasted approximately $2 \mathrm{~h}$ and took place in a quiet classroom on the military installation. Each participant was seated approximately $57 \mathrm{~cm}$ from the assessment PC laptop on which stimuli were presented via E-prime (Version 2.0). 


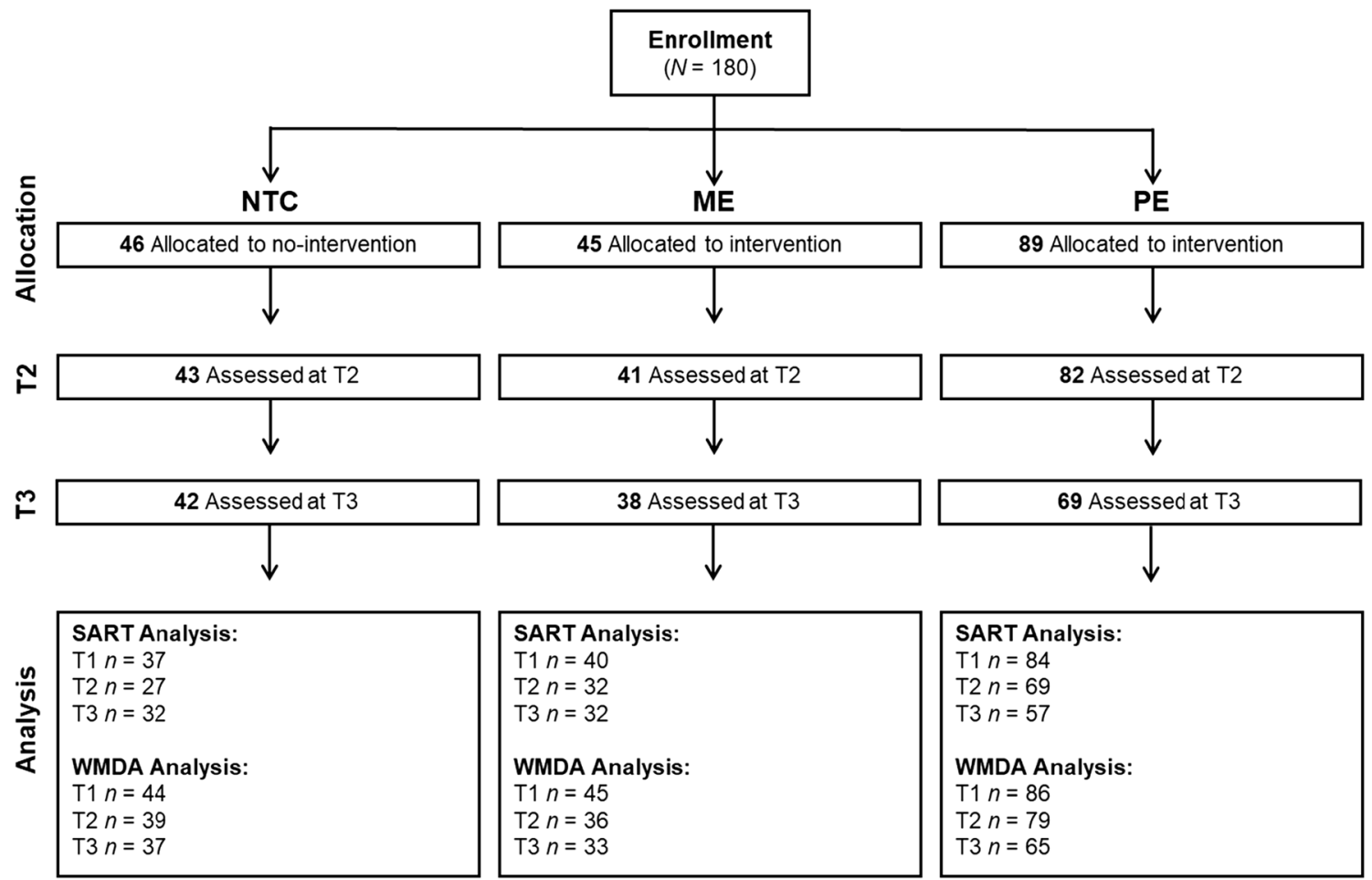

Fig. 1 Consolidated Standards of Reporting Trials Flow Diagram (CONSORT; Schulz et al. 2010) depicting the participation of participants in each study phase and the final sample size included in analyses

Sustained Attention to Response Task (SART) During the SART (Robertson et al. 1997), single digits (0 through 9) were continuously presented on the screen, one at a time, for $250 \mathrm{~ms}$, with each digit followed by an inter-trial interval of $900 \mathrm{~ms}$ during which a fixation cross was presented.
Participants were instructed to refrain from pressing the spacebar in response to the number 3 (target) and to press the spacebar for all other digits (non-targets), while emphasizing both accuracy and speed. Responses were recorded during the stimulus display and the inter-trial interval. Targets

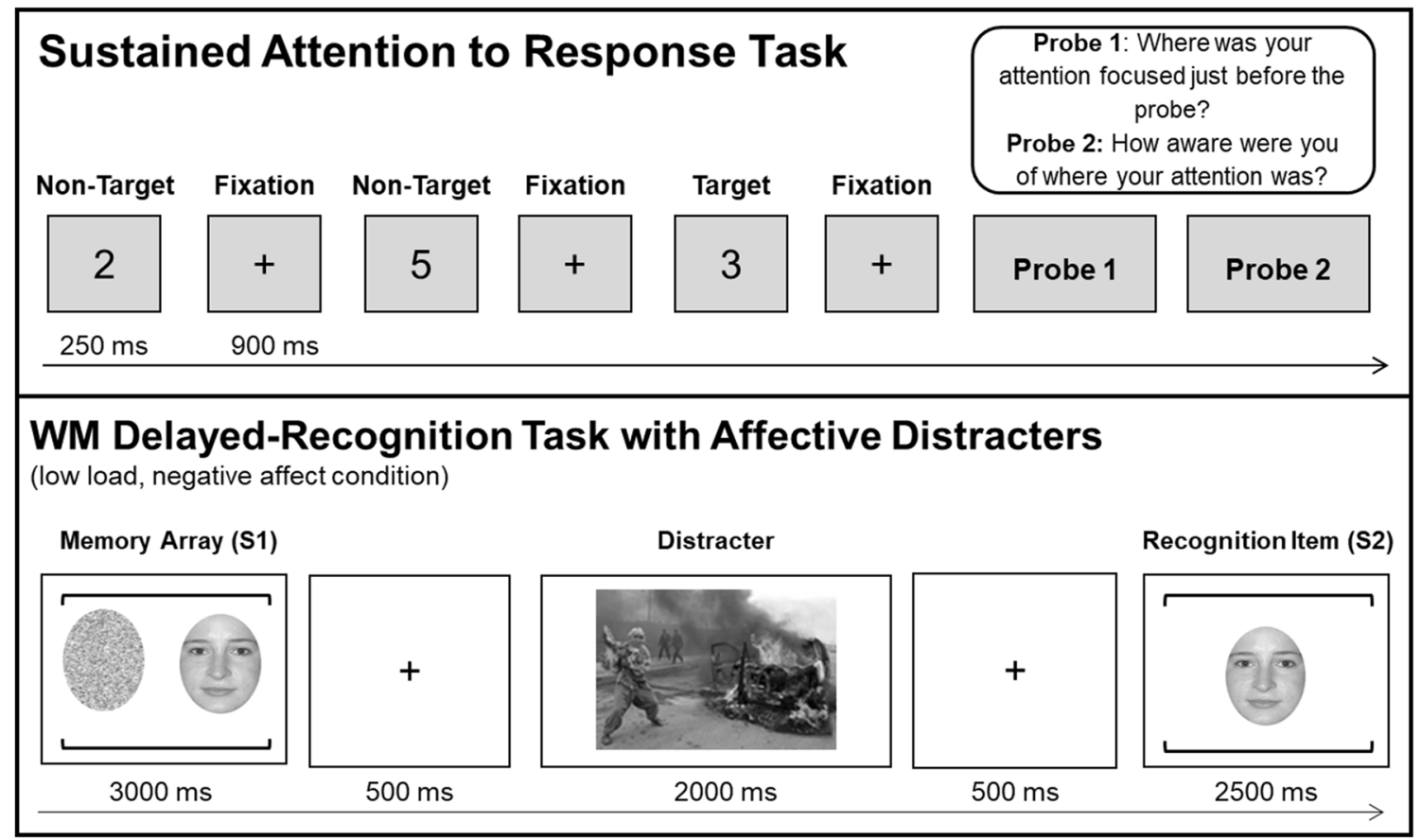

Fig. 2 Schematic depiction of the Sustained Attention to Response Task (SART) and the WM Delayed-Recognition Task with Affective Distracters (WMDA) 
comprised $5 \%$ of trials and non-targets $90 \%$ of trials, and trial order was quasi-randomized so that targets were always separated by at least one other non-target digit.

Participants also responded to two consecutive probe questions, which were dispersed throughout the task (the remaining $5 \%$ of trials) to assess self-reported mind wandering and meta-awareness (Smallwood and Schooler 2015). The first probe (Probe 1) asked, "Where was your attention focused just before the probe?" with participants responding using a 6-point Likert scale ranging from 1 (on task) to 6 (off task). The second probe (Probe 2) asked, "How aware were you of where your attention was?" with participants responding from 1 (aware) to 6 (unaware). The questions were displayed until a response was provided.

After a 163-trial practice block, participants completed two experimental blocks, which consisted of a total of 519 nontargets, 27 targets, and 28 sets of probes. Results from the practice block were not included in the analyses. SART outcomes included task accuracy indexed by $A^{\prime}$, which is a nonparametric measure of sensitivity (see Stanislaw and Todorov 1999 for calculations), and subjective probe responses were indexed using the mean of probe ratings, separately for each probe question.

Working Memory Delayed-Recognition Task with Affective Distracters (WMDA) WM was assessed using a delayedrecognition task instructing participants to remember faces or shoes over a delay interval. This task is the same as one used in previous MT studies of WM (Jha et al. 2017; Zanesco et al. 2019).

Trials began with the encoding phase during which a memory array (S1) containing either two memory items (highmnemonic load) or one memory item paired with a noise mask (low-mnemonic load) was presented for $3000 \mathrm{~ms}$. S1 was followed by a delay interval of $3000 \mathrm{~ms}$, after which a test item (S2) was presented for up to $2500 \mathrm{~ms}$. On half of the trials, S2 was a single image that appeared in S1 (match trials), while on the remaining trials, S2 was a novel image (nonmatch trials) that did not appear in S1 or elsewhere in the experiment. S2 was always of the same category as S1 (face or shoe). Participants were instructed to determine whether S2 matched either memory item in S1 and indicate a match or non-match response by pressing a designated key. Participants were instructed to respond quickly and accurately. Half of the trials utilized faces as stimuli, and the other half utilized shoes, with both trial types intermixed throughout the task.

During the delay interval, a task-irrelevant distracter was displayed for $2000 \mathrm{~ms}$ and was preceded and followed by a fixation cross for $500 \mathrm{~ms}$. On half of the trials, the distracters were negatively valenced; on the other half of trials, they were neutrally valenced. Instructions at the beginning of the task directed participants to keep their gaze in the center of the screen at all times. The distracters were drawn from a previous study conducted in military populations (Morey et al. 2008). The negative distracters were generated from internet searches and photo collections of soldiers that depicted combat-related scenes from Afghanistan and Iraq, while the neutral distracters depicted civilian scenes that matched the negative stimuli in terms of figure/scene ratio, scene complexity, and chromatic structure. Memory items (face or shoe stimuli) and distracter images were not repeated across trials. The task consisted of a 36-trial practice block (with accuracy feedback for the first 6 trials) and two 30-trial experimental blocks.

Task demands were manipulated along two levels of mnemonic load (low vs. high) and two levels of distracter valence (neutral vs. negative), yielding four distinct trial types that were used for analysis: low load-neutral distracter, low loadnegative distracter, high load-neutral distracter, and high loadnegative distracter. Trial order was pseudo-randomly intermixed along these four variables so that identical trial types were never consecutively presented. Accuracy (\% correct) was calculated for each individual for each of the experimental trial types. Trials in which the participant did not respond were excluded from these calculations.

Assessment of Trainer Effectiveness and Out-of-Class MT Practice Compliance To obtain Soldiers' evaluation of trainer effectiveness, participants answered a series of questions at T2. For three questions, participants were asked to respond with one of the following responses: "Not at all," "Somewhat," "Moderately," and "Very." The questions were as follows: (1) "How effective do you think the trainer was at conveying the course material?"; (2) "How effective do you think the trainer was at leading group discussions?"; and (3) "How effective do you think the trainer was at making the material relevant to military life?". Participants were also asked to complete the statement, "Overall, I would rate the trainer's performance as..." with one of the following responses: "Very Poor," "Poor," "Fair," "Good," "Very Good," and "Exceptional." Values (0-3 and 0-5, respectively) were assigned to each response.

Out-of-class mindfulness practice compliance was also assessed during the T2 and T3 testing sessions by asking participants to report the average number of days per week they spent practicing during the 4-week training interval (at T2), and the interval between T2 and T3 (at T3).

\section{Data Analyses}

Change in cognitive task performance over time was evaluated using multi-level linear mixed-effects models implemented in PROC Mixed in SAS 9.4. We examined the fixed effects of time (T1, T2, and T3) and group (NTC, ME, and $\mathrm{PE})$ for each dependent measure. Dependent measures for SART were $A^{\prime}$ and probe responses, and for WMDA, analyses were conducted on \% correct scores. Random intercepts 
Table 2 Descriptive statistics for SART and WM performance

\begin{tabular}{lllll}
\hline Group & Measure & T1 & T2 & T3 \\
\hline NTC & SART $(N)$ & 37 & 27 & 32 \\
& $A^{\prime}$ & $0.816(0.099)$ & $0.722(0.116)$ & $0.720(0.117)$ \\
& Probe 1 & $2.109(1.531)$ & $2.476(1.507)$ & $2.221(1.285)$ \\
& Probe 2 & $1.736(1.205)$ & $2.529(1.459)$ & $2.211(1.207)$ \\
& WMDA $(N)$ & 44 & 39 & 37 \\
& Accuracy & $85.779(7.769)$ & $81.913(11.857)$ & $79.092(12.293)$ \\
ME & SART $(N)$ & 40 & 32 & 32 \\
& $A^{\prime}$ & $0.792(0.114)$ & $0.712(0.149)$ & $0.686(0.140)$ \\
& Probe 1 & $1.779(1.022)$ & $2.860(1.716)$ & $2.190(1.738)$ \\
& Probe 2 & $1.695(0.808)$ & $2.826(1.575)$ & $2.224(1.774)$ \\
& WMDA $(N)$ & 45 & 36 & 33 \\
& Accuracy & $86.227(6.959)$ & $78.845(13.008)$ & $76.618(12.509)$ \\
PE & SART $(N)$ & 84 & 69 & 57 \\
& $A^{\prime}$ & $0.784(0.130)$ & $0.774(0.136)$ & $0.720(0.135)$ \\
& Probe 1 & $1.969(1.212)$ & $2.263(1.388)$ & $2.327(1.331)$ \\
& Probe 2 & $1.899(1.176)$ & $2.262(1.396)$ & $2.199(1.321)$ \\
& WMDA $(N)$ & 86 & 79 & 65 \\
& Accuracy & $86.665(8.127)$ & $84.792(9.910)$ & $81.763(13.072)$ \\
& &
\end{tabular}

Mean $(S D)$ values are provided for $A^{\prime}$ and subjective probe responses in the SART and overall accuracy (\% correct) in the WMDA task for each group (NTC, ME, and PE) before (T1) and after (T2) the 4-week training interval, and again after training following a 4-week interval (T3). The number of participants $(N)$ is provided for each task and assessment

were included for individuals, representing between-person variability, and separate residual variances were estimated for each of the four companies (NTC, $\mathrm{ME}, \mathrm{PE}_{1}$, and $\mathrm{PE}_{2}$ ) to accommodate heterogeneity in company variance. Individuals were not hierarchically nested within companies; however, as less than $1 \%$ of variance was accounted for by clustering at the company-level based on the intra-class correlation calculated from the null-model for all dependent measures. Parameters from all models were estimated using maximum likelihood estimation, and degrees of freedom were approximated by dividing the residual degrees of freedom into between-person and within-person divisions. Model parameters were referenced to T1 and the NTC group. For analyses of WMDA task performance, parameters were further referenced to the low load and neutral valence conditions. Type III tests of fixed effects are reported alongside parameter estimates for all models.

Multi-level linear mixed models allow for the inclusion of participants with missing data at one or more assessment wave minimizing prognostic bias in estimates of treatment effects attributed to dropout rates, and increasing statistical power by preserving greater sample size (Gupta 2011). We, therefore, included all participants in our analyses who contributed data on a particular dependent measure regardless of dropout or missing data. We did, however, exclude observations from participants if they did not comply with proper task instructions, or represented statistical outliers (i.e., for SART, performance below chance, $A^{\prime}<0.5$; for WMDA, performance $3 \mathrm{SD}$ below the grand mean; see Zanesco et al. 2019). There were 175 participants (Obs $n=410$ ) in total contributing to the analyses of SART dependent measures, and 179 participants (Obs $n=1860$ ) in total contributing to the analyses of WMDA. Figure 1 summarizes the number of participants at
Table 3 Parameter estimates from analyses of SART performance

\begin{tabular}{lccc}
\hline & \multicolumn{2}{l}{ Estimate $(\mathrm{SE})$} & \\
\cline { 2 - 4 } Model effects & Accuracy $\left(A^{\prime}\right)$ & Probe 1 & Probe 2 \\
\hline Fixed effects & & & \\
Intercept & $0.804(0.021)^{* * *}$ & $2.047(0.239)^{* * *}$ & $1.668(0.199)^{* * *}$ \\
Time 2 & $-0.103(0.024)^{* * *}$ & $0.392(0.302)$ & $0.787(0.232)^{* * *}$ \\
Time 3 & $-0.099(0.022)^{* * *}$ & $0.181(0.287)$ & $0.574(0.221)^{* * *}$ \\
ME group & $-0.019(0.029)$ & $-0.206(0.325)$ & $0.077(0.288)$ \\
PE group & $-0.023(0.025)$ & $-0.066(0.279)$ & $0.236(0.246)$ \\
ME group $\times$ time 2 & $0.021(0.031)$ & $0.700(0.399)$ & $0.314(0.340)$ \\
ME group $\times$ time 3 & $0.005(0.031)$ & $0.322(0.389)$ & $0.016(0.334)$ \\
PE group $\times$ time 2 & $0.077(0.027)^{* *}$ & $-0.085(0.342)$ & $-0.383(0.287)$ \\
PE group $\times$ time 3 & $0.023(0.027)$ & $0.301(0.335)$ & $-0.174(0.286)$ \\
Random effects & & & \\
Intercept variance & 0.009 & 0.839 & 0.741 \\
NTC residual variance & 0.008 & 1.324 & 0.768 \\
ME residual variance & 0.007 & 1.131 & 1.027 \\
PE residual variance & 0.006 & 0.787 & 0.916 \\
PE residual variance & 0.008 & 1.108 & 1.179 \\
-2 Log-likelihood & -619.4 & 1368.8 & 175 \\
Participants $(N)$ & 175 & 175 & 410 \\
Observations & 410 & 410 & 10.7 \\
\hline
\end{tabular}

Maximum likelihood estimates are reported for models of SART accuracy $\left(A^{\prime}\right)$, and mean probe 1 and 2 ratings, for fixed effects of time (T1, T2, and T3) and group (NTC, ME, and PE). T1 and NTC group serve here as the reference condition. Separate residual variances are estimated for each company. The number of participants $(N)$ and total observations contributing to the analyses are provided. Standard errors are reported in parentheses $* p<0.05 ; * * p<0.01 ; * * p<0.001$ 
each assessment wave and the total number of observations contributing to analyses of each dependent measure.

Additional analyses performed without any exclusions are reported in the Electronic Supplemental Material.

\section{Results}

Table 2 provides descriptive statistics for dependent measures derived from the SART and WMDA for each group and assessment.

\section{Sustained Attention to Response Task (SART)}

The primary analyses for the SART $(n=175)$ were conducted with separate mixed-effects models for each outcome variable ( $A^{\prime}$ and subjective probe responses) comparing groups (NTC, $\mathrm{ME}$, and PE) over time (T1, T2, and T3). Comparisons between all individual group cohorts (NTC, $\mathrm{ME}, \mathrm{PE}_{1}$, and $\mathrm{PE}_{2}$ groups) are reported in the Electronic Supplemental Material.

\section{A' Scores}

There was a significant effect of time, $F(2,229)=35.36$, $p<0.001$, no significant effect of group, $F(2,172)=0.51$, $p=0.600$, and a significant interaction of time and group, $F(4,229)=2.55, p=0.040$. Parameter estimates from this model are provided in Table 3 and discussed below.

At the onset of the study (T1), both the ME $(b=-0.019$, $p=0.512,95 \%$ CI $[-0.076,0.038])$ and PE groups $(b=-$ $0.023, p=0.361,95 \%$ CI $[-0.073,0.027])$ did not significantly differ from the NTC group. The NTC group declined in $A^{\prime}$ from T1 to T2 $(b=-0.103, p<0.001,95 \%$ CI $[-0.149,-$ $0.057])$ and from T1 to T3 ( $b=-0.099, p<0.001,95 \%$ CI [$0.143,-0.055])$. The ME group also declined in $A^{\prime}$ from $\mathrm{T} 1$ to T2 $(b=-0.082, p<0.001,95 \%$ CI $[-0.123,-0.041])$ and from T1 to T3 $(b=-0.095, p<0.001,95 \%$ CI $[-0.136,-$ 0.053]). Importantly, however, the PE group did not significantly decline in $A^{\prime}$ from T1 to T2 $(b=-0.026, p=0.064$, $95 \%$ CI $[-0.054,0.002])$ but did decline from T1 to T3 $(b=-0.076, p<0.001,95 \%$ CI $[-0.106,-0.046])$.

These patterns suggest that the PE group changed differently compared to the NTC and ME groups. Relative to the NTC group, the PE group declined significantly less from T1 to T2 ( $b=0.077, p=0.006,95 \%$ CI $[0.023,0.131])$, but did not change differently from T1 to T3 $(b=0.023, p=0.390$, $95 \%$ CI $[-0.030,0.077])$. Relative to the ME group, the PE group also declined significantly less from T1 to T2 $(b=$ $0.056, p=0.027,95 \%$ CI $[0.006,0.105])$, but did not change differently from T1 to T3 $(b=0.019, p=0.468,95 \%$ CI [$0.032,0.070])$. When compared to the NTC group, the ME group did not change differently from $\mathrm{T} 1$ to $\mathrm{T} 2(b=0.021$, $p=0.507,95 \%$ CI $[-0.041,0.083])$ nor from T1 to T3 $(b=$
$0.005, p=0.883,95 \%$ CI $[-0.056,0.065])$. Observed participant data, model estimates of means, and the change in SART $A^{\prime}$ are depicted in Fig. 3 for each group. Thus, examination of the significant time $\times$ group interactions for SART $A^{\prime}$ scores revealed that $\mathrm{PE}$-trained participants were best protected against performance decline over time relative to the NTC and ME groups.

\section{Subjective Probe Responses}

For scores on probe 1, we observed a significant effect of time, $F(2,229)=9.16, p<0.001$; no significant effect of group, $F(2,172)=0.20, p=0.815$; and no significant interaction of time and group, $F(4,229)=2.20, p=0.069$. Parameter estimates from this model are provided in Table 3. Overall, groups reported being significantly more off task from T1 to T2 $(b=0.597, p<0.001,95 \%$ CI $[0.315,0.880])$ and from T1 to T3 ( $b=0.389, p=0.007,95 \%$ CI $[0.109,0.668])$.

For scores on probe 2 , we observed a significant effect of time, $F(2,229)=19.48, p<0.001$, no significant effect of group, $F(2,172)=0.35, p=0.705$, and no significant interaction of time and group, $F(4,229)=1.49, p=0.207$ (see Table 3 for parameter estimates). Again, groups reported being significantly more unaware of their attentional state from T1 to T2 $(b=0.764, p<0.001,95 \%$ CI $[0.514,1.013])$ and from T1 to T3 $(b=0.522, p<0.001,95 \%$ CI $[0.272,0.771])$.

\section{Working Memory Delayed-Recognition Task with Affective Distracters}

Analyses for WM $(n=179)$ were conducted with mixedeffects models examining within-subject factors of mnemonic load (high and low), distracter valence (negative and neutral), and time (T1, T2, and T3), and a between-subjects factor of group (NTC, ME, and PE) on WM task performance (\% correct). Comparisons between all individual group cohorts (NTC, $\mathrm{ME}, \mathrm{PE}_{1}$, and $\mathrm{PE}_{2}$ groups) are reported in the Electronic Supplemental Material.

\section{Accuracy (\% Correct)}

We observed a significant effect of load, $F(1,176)=296.73$, $p<0.001$, valence, $F(1,176)=129.16, p<0.001$, and a significant interaction of load and valence, $F(1,176)=13.04$, $p<0.001$. Furthermore, there was a significant effect of group, $F(2,176)=4.09, p=0.018$, a significant effect of time, $F(2,280)=63.20, p<0.001$, and a significant interaction of time and group $F(4,280)=7.29, p<0.001$. There were no other significant interactions between load, valence, group, or time (all $p \mathrm{~s}>0.232$ ). Accordingly, we examined the significant effects of load, valence, and their interaction, together with effects of time, group, and their interaction, in a 
Fig. 3 Observed participant data are shown (a) across assessments for SART $\left(A^{\prime}\right)$, separately for the NTC, ME, and PE groups. The model estimated means derived from mixed-effects models are depicted (b) for groups. Error bars represent $95 \%$ confidence intervals surrounding the estimate. Parameter estimates of the change (c) from $\mathrm{T} 1$ to $\mathrm{T} 2$ and change (d) from T1 to T3 for the NTC, ME, and $\mathrm{PE}$ groups are also given. Error bars represent $95 \%$ confidence intervals surrounding the model estimated change. $* p<0.05, * * p<0.01$ $* * * p<0.001$
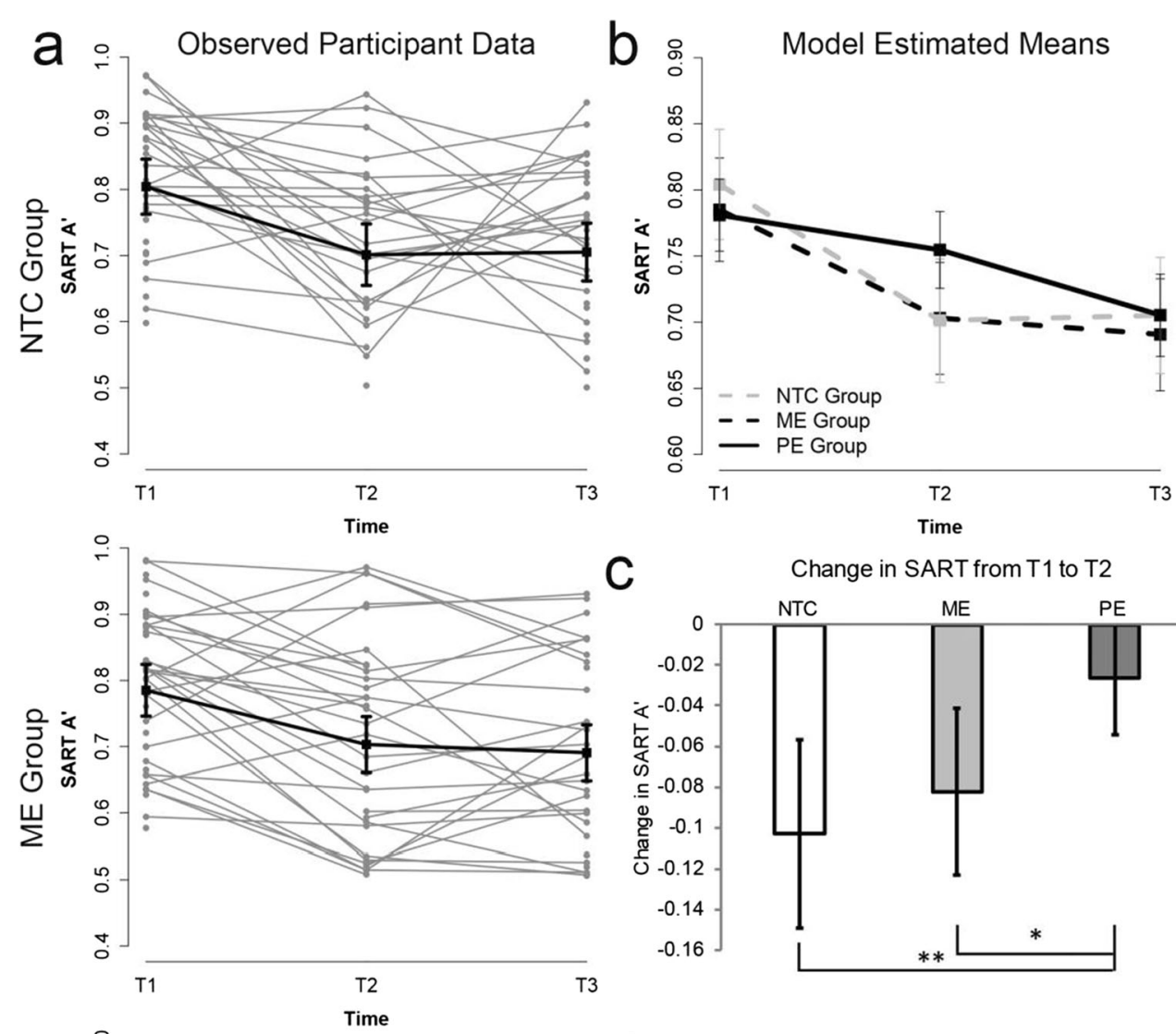

C Change in SART from T1 to T2
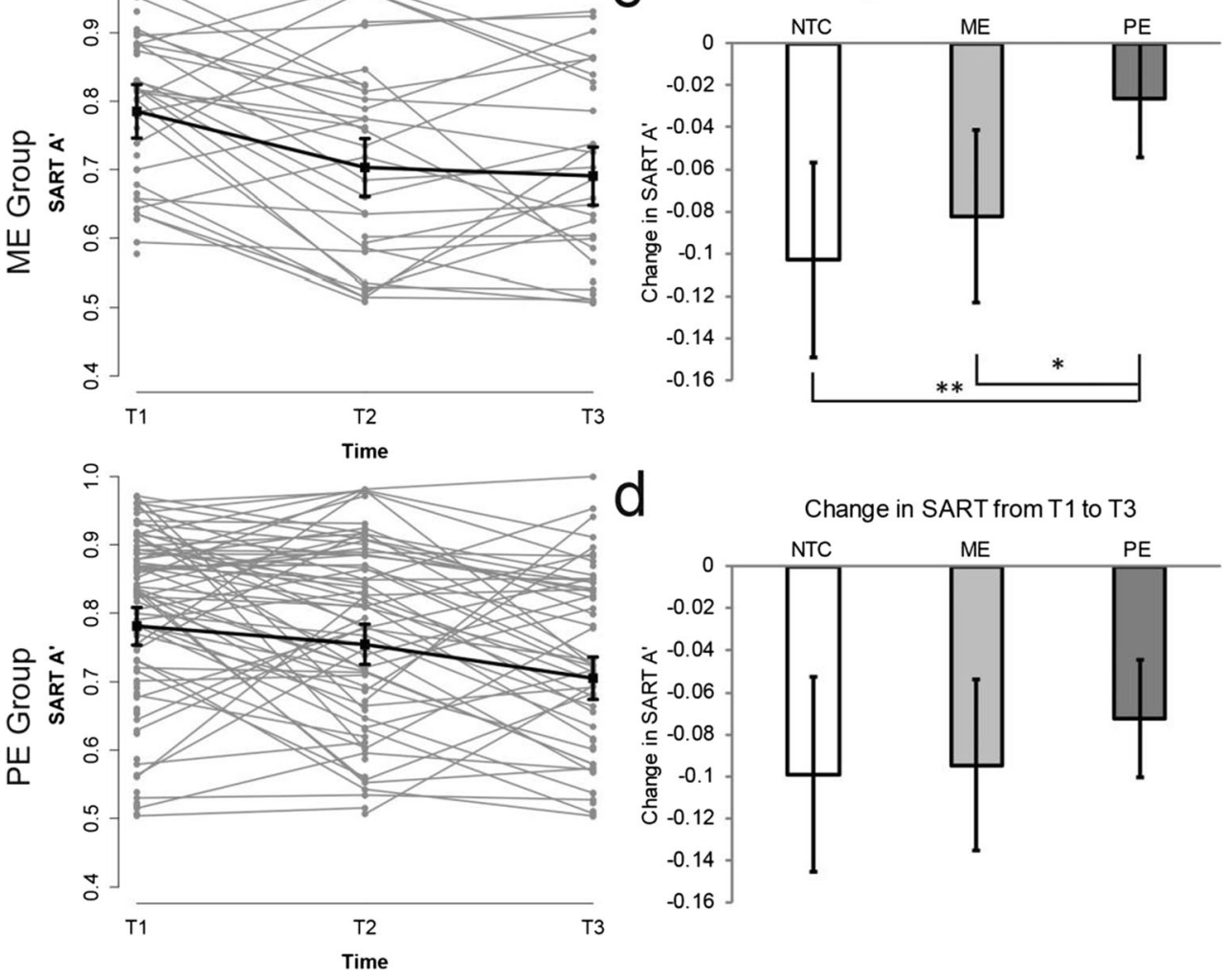

simplified model. Parameter estimates from this model are provided in Table 4 and discussed below.

Accuracy was lower when load was high $(b=-7.069 \%$, $p<0.001,95 \%$ CI $[-8.397,-5.741])$, lower when interference was negative $(b=-4.047 \%, p<0.001,95 \%$ CI $[-5.375$, $-2.719]$ ), and further influenced when these demands combined on trials $(b=-4.071 \%, p<0.001,95 \%$ CI $[-5.949$, $2.193])$. These load and valence effects are consistent with prior use of this task (Jha et al. 2017; Zanesco et al. 2019).

At T1, both the ME $(b=0.377 \%, p=0.854,95 \%$ CI [$3.668,4.423])$ and $\mathrm{PE}$ groups $(b=0.229 \%, p=0.896,95 \%$ CI $[-3.221,3.679])$ did not significantly differ from the NTC group. The NTC group declined in overall WM accuracy from T1 to T2 $(b=-3.939 \%, p=0.002,95 \%$ CI $[-6.450,-$
$1.429])$, and from T1 to T3 $(b=-7.221 \%, p<0.001,95 \% \mathrm{CI}$ $[-9.763,-4.679])$. The ME group also declined in overall WM accuracy from T1 to T2 $(b=-8.447 \%, p<0.001,95 \%$ CI $[-11.044,-5.851])$ and from T1 to T3 $(b=-10.845 \%$, $p<0.001,95 \%$ CI $[-13.497,-8.193])$. Importantly, however, the PE group did not significantly decline in overall WM accuracy from T1 to T2 $(b=-1.378 \%, p=0.065,95 \%$ CI [$2.844,0.089])$, but did decline from T1 to T3 $(b=-4.153 \%$, $p<0.001,95 \%$ CI $[-5.737,-2.569])$.

These patterns suggest that the PE group changed differently compared to the NTC and ME groups. Relative to the NTC group, the PE group did not change significantly differently from $\mathrm{T} 1$ to $\mathrm{T} 2(b=2.561 \%, p=0.084,95 \% \mathrm{CI}[-0.346$, 5.469]) but declined significantly less from $\mathrm{T} 1$ to $\mathrm{T} 3(b=$ 
Table 4 Parameter estimates from analysis of WMDA accuracy

\begin{tabular}{lc}
\hline Model effects & Estimate $(S E)$ \\
\hline Fixed effects & \\
Intercept & $92.425(1.508)^{* * *}$ \\
Load & $-7.069(0.673)^{* * *}$ \\
Valence & $-4.047(0.673)^{* * *}$ \\
Load $\times$ valence & $-4.071(0.952)^{* * *}$ \\
Time 2 & $-3.939(1.275)^{* *}$ \\
Time 3 & $-7.221(1.291)^{* * *}$ \\
ME group & $0.377(2.050)$ \\
PE group & $0.229(1.748)$ \\
ME group $\times$ time 2 & $-4.508(1.835)^{*}$ \\
ME group $\times$ time 3 & $-3.624(1.866)$ \\
PE group $\times$ time 2 & $2.561(1.477)$ \\
PE group $\times$ time 3 & $3.068(1.521)^{*}$ \\
Random effects & \\
Intercept variance & 61.391 \\
NTC residual variance & 128.500 \\
ME residual variance & 131.870 \\
PE 1 residual variance & 85.383 \\
PE 2 residual variance & 91.164 \\
2 Log-likelihood & $14,318.5$ \\
Participants $(N)$ & 179 \\
Observations & 1860 \\
\hline
\end{tabular}

Maximum likelihood estimates are reported for models of WMDA accuracy (\% correct) for fixed effects of time (T1, T2, and T3) and group (NTC, ME, and PE). T1 and NTC group serve here as the reference condition. Load and valence are referenced to the low-load neutral condition. Separate residual variances are estimated for each company. The number of participants $(N)$ and total observations contributing to the analyses are provided. Standard errors are reported in parentheses

$* p<0.05 ; * * p<0.01 ; * * * p<0.001$

$3.068 \%, p=0.045,95 \%$ CI $[0.073,6.063])$. Furthermore, compared to the ME group, the PE group declined significantly less from T1 to T2 $(b=7.070 \%, p<0.001,95 \%$ CI $[4.088,10.051])$ and from T1 to T3 $(b=6.692 \%, p<0.001,95 \%$ CI [3.603, 9.781]). When compared to the NTC group, the ME group declined significantly more from $\mathrm{T} 1$ to $\mathrm{T} 2(b=-4.508 \%, p=$ $0.015,95 \%$ CI $[-8.120,-0.897])$, but did not change significantly differently from $\mathrm{T} 1$ to $\mathrm{T} 3$ ( $b=-3.624 \%, p=0.053,95 \%$ CI [-7.297, 0.049]). Observed participant data and model estimates of means, as well as change in accuracy are depicted in Fig. 4 for each group.

These findings revealed that PE-trained participants were best protected against WM performance decline over time relative to the NTC and ME groups. The protective effects were most pronounced in the $\mathrm{T} 1$ to $\mathrm{T} 3$ interval for the $\mathrm{PE}$ group. They experienced less of a reduction in their WM accuracy compared to the NTC and ME groups over the 10week study interval.

\section{Participants' Perceptions of Trainer Effectiveness and Out-of-Class MT Practice Compliance}

\section{Participants' Perceptions of Trainers}

Soldiers' ratings of their trainer at T2 ( $\mathrm{PE} n=82$ and $\mathrm{ME} n=$ 40 ) were compared across groups with independent samples $t$ tests. The PE trainers (compared to the ME trainer) were rated as being significantly more effective at conveying the course material, leading group discussions, contextualizing the material for military life, and they were rated as more effective overall (all $p \mathrm{~s}<0.017$; see Table 5 for descriptive statistics and independent samples $t$-tests). Standardized effect sizes are calculated as Hedges $g$ (Lakens 2013). Moderate to large differences ( $g$ range $=0.528-1.145$ ) in participants' perceptions of trainer effectiveness were, therefore, present between the ME and PE groups.

\section{Self-Reported Out-of-Class MT Practice Compliance}

Soldiers in the PE group $(n=82)$ reported practicing an average of 2.48 days per week out-of-class $(S D=2.04)$ during the training interval when asked at $\mathrm{T} 2$, whereas the ME group $(n=40)$ reported practicing an average of 1.55 days per week $(S D=1.91)$. For the interval between T2 and T3, PE soldiers $(n=65)$ reported practicing an average of 3.09 days per week $(S D=2.65)$, while the ME group $(n=37)$ reported practicing an average of 1.19 days per week. Independent samples $t$ tests revealed that the PE group practiced more days per week on average than the ME group, $t(82.47)=2.46, p=0.016, g=$ 0.460 , during the training interval, and more days on average than the ME group, $t(89.68)=4.00, p<0.001, g=0.767$, in the interval between T2 and T3. Thus, Soldiers in the PE group practiced more frequently across the entire study interval than did the ME group.

\section{Discussion}

The broad aim of the current study was to determine successful dissemination pathways for MT in naturalistic military settings. We investigated train-the-trainer (TTT) delivery of a short-form ( $8 \mathrm{~h})$ MT program, called MBAT, to activeduty soldiers over a high-demand interval. PEs, who were "in house" context-familiar trainers but naïve to MT at the outset of the project, were selected as trainers to deliver MBAT to Soldiers. The present findings revealed that while task performance declined over the high-demand military field training interval for all participants, the PE group showed less decline when compared to the NTC group, as well as the ME group.

In line with prior studies reporting cognitive vulnerabilities over high-demand military training intervals (e.g., Lieberman 
Fig. 4 Observed participant data are shown (a) across assessments for WMDA accuracy (\% correct), separately for the NTC, ME, and PE groups. The model estimated means derived from mixedeffects models are depicted (b) for groups. Error bars represent 95\% confidence intervals surrounding the estimate. Parameter estimates of the change (c) from $\mathrm{T} 1$ to $\mathrm{T} 2$ and change (d) from $\mathrm{T} 1$ to $\mathrm{T} 3$ for the NTC, ME, and PE groups are also given. Error bars represent $95 \%$ confidence intervals surrounding the model estimated change. ${ }^{*} p<0.05, * * p<0.01$, $* * * p<0.001$
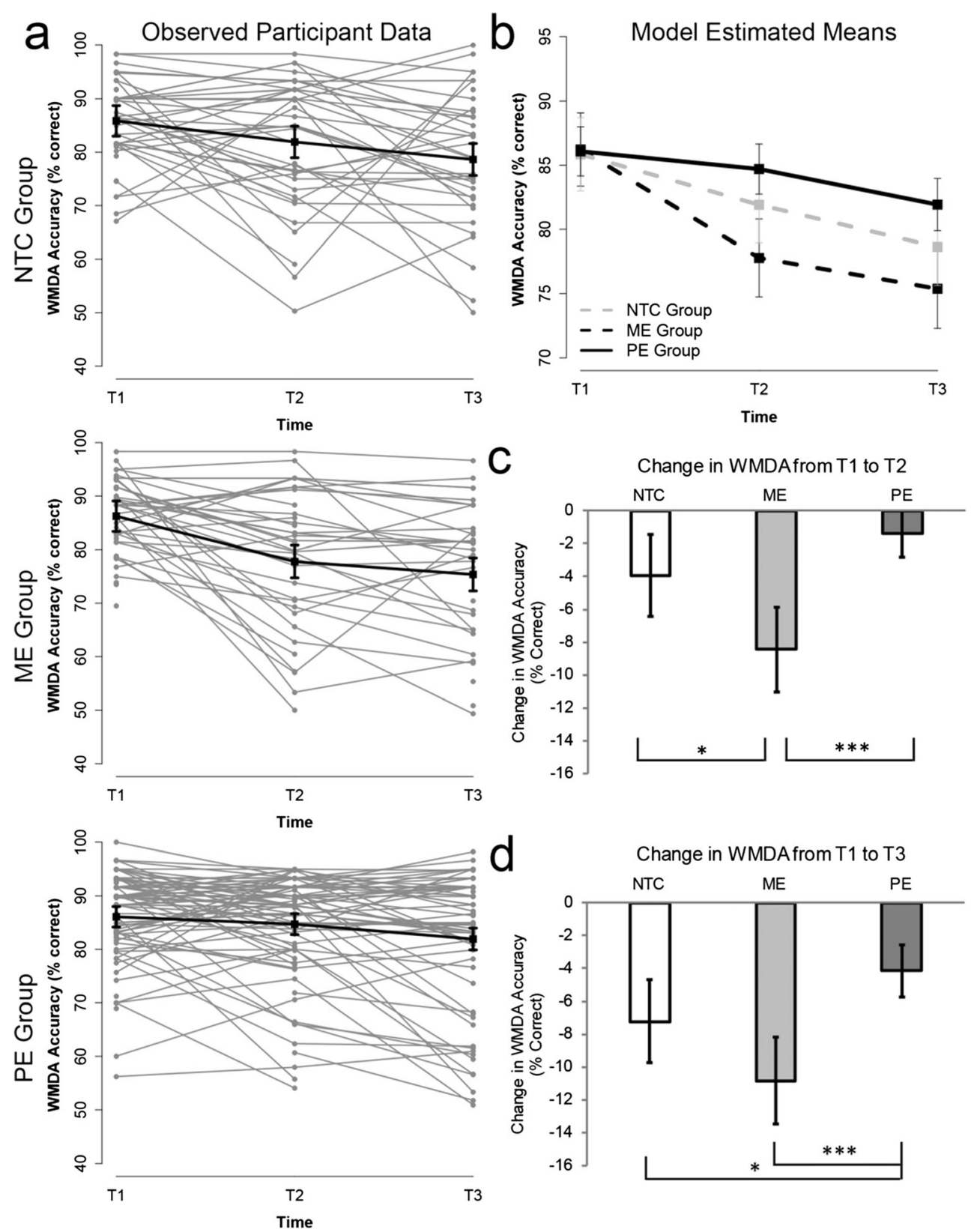

et al. 2005), task performance on the SART and the WMDA task declined over the study interval (i.e., T1 to T3) in all participants. Indeed, for the SART, across all groups, $A^{\prime}$ was lower, and probe responses indicated that Soldiers had greater
Table 5 Descriptive statistics of trainee ratings of trainer effectiveness

\begin{tabular}{llllll}
\hline & ME & PE & $t$ value $(d f)$ & $p$ value & $g$ \\
\hline 1. Conveying material & $1.70(1.22)$ & $2.52(0.69)$ & $3.97(51.40)$ & $<0.001$ & 0.908 \\
2. Leading discussion & $1.53(1.13)$ & $2.51(0.79)$ & $4.96(58.13)$ & $<0.001$ & 1.065 \\
3. Contextualizing material & $0.88(1.02)$ & $2.04(1.00)$ & $5.95(76.21)$ & $<0.001$ & 1.145 \\
4. Overall performance & $3.15(1.61)$ & $3.85(1.15)$ & $2.48(58.90)$ & 0.016 & 0.528 \\
\hline
\end{tabular}

Mean $(S D)$ values are provided for trainee ratings of trainer effectiveness at the end of training (T2) for the ME and PE groups. Questions 1-3 were rated by participants from 0 (not at all effective) to 3 (very effective), and question 4 was rated from 0 (very poor) to 5 (very good). Independent samples $t$ tests $(d f), p$ values, and standardized effect sizes (Hedges' $g$ ) are provided. Degrees of freedom $(d f)$ were adjusted to account for inequality of variances 
off-task thought (greater mind wandering, probe 1) and were less aware of their own attentional state (less meta-awareness, probe 2) at $\mathrm{T} 3$ relative to $\mathrm{T} 1$. In addition, overall WMDA task accuracy (\% correct) was lower by the end of the 10 -week study interval. Thus, sustained attention and WM performance declined, mind wandering increased, and meta-awareness decreased over the high-demand military field training interval.

Worsening performance over intensive intervals of predeployment training has been previously reported for both the SART (Jha et al. 2015, 2016) and the WMDA task (Jha et al. 2010, 2017). Such changes in task performance are unlikely to be due to task instability, since task performance has been shown to remain stable in civilian cohorts over an 8-week interval of typical civilian life (e.g., Jha et al. 2015; Jha et al. 2017). We suggest that the changes in SART and WMDA task metrics observed herein may reflect depletion of cognitive resources that are used in the service of persistent cognitive and emotional challenges soldiers face during protracted military training (see Jha et al. 2016, for discussion). Nonetheless, additional research is needed to uncover the precise mechanisms involved in cognitive depletion over time in military cohorts. For example, little is known about the specific relationships between cognitive decline and physiological metrics of stress (e.g., Lieberman et al. 2005).

We examined if PE-delivered MBAT protected against such a decline relative to the NTC group. Indeed, the PEtrained group demonstrated significantly less decline than the NTC group from T1 to T2 for SART performance. Specifically, the PE group was estimated to decline less compared to the NTC group by 0.08 units of $A^{\prime}$ in the SART from T1 to T2, which represent roughly 8 fewer missed SART target trials (in a participant with 0 non-target errors). Such a relative performance advantage on the SART may have farreaching consequences, as the SART has been considered a valid model for friendly-fire incidents (Wilson et al. 2015). Yet, we found only directional but not significant differences between the PE and NTC groups in the amount of attentional change from $\mathrm{T} 1$ to $\mathrm{T} 3$, suggesting that the attentional benefits were not completely maintained across the entire study interval (10 weeks in total). Future studies will need to explore the factors that contribute to the longer-term maintenance of MTrelated benefits in high-demand settings beyond the formal period of trainer-led training, perhaps by facilitating continued MT practice in participants.

Interestingly for the WMDA task, PE-trained participants showed significantly less decline than NTC from T1 to T3, but only a directional difference from $\mathrm{T} 1$ to $\mathrm{T} 2$. Specifically, the PE group was estimated to decline less compared to the NTC group by $3.07 \%$ accuracy in the WMDA task from T1 to T3, which represents roughly 2 fewer incorrect WMDA trials on average. Such a performance advantage for PE-trained participants vs. NTC was roughly equivalent to the observed cost of negative affective distracters on WM accuracy. One question is why we observed these benefits over the entire 10 -week interval, but not as robustly immediately after completion of the MBAT program. One possibility is that while attention and WM are interrelated (e.g., Jha 2002), and amenable to being strengthened with MT (e.g., Jha et al. 2015, 2017), they may follow slightly different trajectories of degradation and/or improvement.

Next, we investigated if MBAT's salutary effects differed based on delivery by PEs vs. the ME. For the SART, consistent with the PE vs. NTC comparison, the PE group declined less than the ME group from T1 to T2 but did not significantly differ from the ME group in the amount of attentional change from T1 to T3. For the WMDA task, the PE group declined less than the ME group from T1 to T2 as well as from T1 to T3. Together, these results suggest that not only was PE-delivered MBAT protective against decline relative to the NTC group, but it was also protective relative to ME-delivered MBAT.

Given that both types of trainers (PEs and ME) delivered the same structured program, assigned the same amount of outof-class mindfulness practice, and received training to prepare them to deliver the MBAT program, what might have driven differences in the magnitude of benefits as a function of trainer type? One possible explanation comes from the examination of Soldiers' ratings of their trainers. Perceptions of trainer effectiveness were higher for the PE vs. ME group for each of the domains of course delivery reported on by trainees (see Table 5). It may be that Soldiers were more willing or more easily able to fully attend to and comprehend the course content when delivered by a trainer they perceived as being effective. With a better understanding of practice instructions, for example, their practice integrity may have been higher. Another, albeit related, possibility is that when trainers were perceived as more effective, Soldiers were more motivated or willing to engage in out-of-class practice as well. Indeed, the mean number of days per week that the PE group practiced was also significantly greater than that of the ME group.

It is also possible that the soldiers were more skeptical of MT when delivered by the ME, given the ME's lack of knowledge about the military context. A similar interpretation was proposed by one TTT study on motivational interviewing (MI) in prisons (Doran et al. 2013). Namely, Doran et al. (2013) suggested that while expert consultants may have been better able to conduct MI, participants were skeptical of these trainers, given their lack of knowledge of the prison environment. Herein, the ME may have been more adept at delivery of MT principles, technique instruction, and execution guidance. Yet, Soldiers' skepticism, driven by their perceptions of the ME's lack of context familiarity regarding the Soldier experience, may have caused them to disengage from the material the trainer was aiming to convey or the discussions being led. This disengagement may have, in turn, contributed to a reduction in out-of-class practice. Collectively, these factors likely contributed to their lower ratings of the ME's 
effectiveness as well as the absence of protective effects on cognitive performance for the ME group.

However, the results herein cannot strongly claim that the results in the ME group were solely driven by the ME's lack of context familiarity. For example, it is possible that other factors may have contributed to our inability to observe protective benefits in the ME group relative to NTC. Indeed, it is possible that other trainer-specific characteristics, such as personality, moderated the present findings. Or perhaps specific characteristics of the company assigned to the ME trainer differed in important, yet-unknown-to-us ways from other companies. Future studies should continue to evaluate trainer-related differences by comparing additional PEs and MEs and by formally assessing trainer efficacy using standardized assessment criteria. Nonetheless, our results support the conclusion that a cost-effective and time-efficient strategy for scalable and rapid dissemination of short-form MT is to train PEs (or other context-familiar persons) to deliver MT.

\section{Limitations and Future Research Directions}

Some limitations in the design of the present study are tied to the challenges associated with studying active-duty military service members. In this population, research participation is secondary to the primary aim of military training and preparation. Our research effort functioned within existing organizational structures and was bound by training schedule constraints. MT randomization occurred based on company-level assignment. Thus, it possible that companies differed systematically along important dimensions (e.g., workload or stress). As such, we cannot rule out the effects of preexisting group differences on our results. Nonetheless, training groups were matched as best as could be accommodated (e.g., age, education, engagement in intensive field training). Furthermore, intervention arms aimed to match for psychosocial support, inand out-of-class time demands, and participant expectations of benefits. Future studies should use larger sample sizes to comprehensively explore a full spectrum of demographic variables, as well as cognitive, affective, personality, and motivational factors that may contribute to the magnitude of benefits observed with MT. In addition, the results of the present study should be replicated in larger samples of troops, with a study design including random assignment at the individual level, and cognitive performance effects measured longitudinally over longer intervals beyond a high-demand field training interval. Such designs, while theoretically appropriate and experimentally ideal, may not be feasible in the applied setting of active-duty military units. Thus, research in this context aims to best accommodate high-research standards while accepting that this is secondary to the military mission.

Another limitation of the current study is that the methodology and results may not generalize to clinical populations. Indeed, the MBAT program was designed for delivery to psychologically and physically healthy individuals in highdemand, time-pressured workplace contexts. MBAT program development was predicated on the need for scalable access to MT by specific groups, such as military service members. While the MBAT training practicum followed well-accepted TTT recommendations for MBPs (Crane et al. 2017), the duration of the foundational and basic training delivery components of the practicum are shorter than what is typical in programs such as MBSR or Mindfulness-Based Cognitive Therapy. Yet, currently accepted MT trainer development pathways are labor intensive and often prohibitively costly (Marx 2019), which can impede scalability and accessibility in offering MT to broader communities (Marx 2019). Nonetheless, we acknowledge that our conclusions may not apply to patient populations or longer-form MT programs, where having trainers who are substantially more adept, familiar, and sophisticated in MT may be critical (Crane et al. 2017). Nonetheless, interest is growing in offering MT to various groups, and a continued research effort is necessary to identify best practices for effective and expeditious training delivery.

In sum, the present study provides preliminary evidence for effectiveness with regard to train-the-trainer delivery of MBAT by PEs in the military context. The relative success of PE- over ME-delivered MBAT suggests that recruiting trainers from the organizational context in which training will be offered may be a fruitful strategy for broader dissemination of MT in applied settings. Trainers that have prior experience training constituents within their organization may be best positioned to learn the additional skills and content in order to deliver MT effectively. Academy instructors at police or firefighter academies, or medical school faculty who teach medical students, or human resources staff members who offer group workshops at companies, are examples of individuals who know their communities well and have prior experience facilitating group training. Future studies should examine MT delivery by such individuals in the service of identifying best practices for MT delivery in applied settings.

Acknowledgements We thank the U.S. Army Resiliency Directorate, Dr. Coreen Harada, and Dr. Amy Adler for their assistance with this project. We thank Dr. Stephen Gonzalez, Nick Fuller, Tyler Pauly, Gus Castellanos, Merissa Goolsarran, Eliot Tang-Smith, and Laura MacKinnon for their assistance with training and data collection, and MBAT advisory board members Lieutenant Gen. Walter Piatt, Col. (R) Michael Brumage, Col. (R) Charles Hogue, Sharon Salzberg, Dr. Jon Kabat-Zinn, and Dr. Michael Gervais for their guidance and input regarding the MBAT program.

Author Contributions APJ conceived of and designed the experiments. APJ and SLR conceived of and developed the mindfulness intervention. SLR and JWG supported implementation and delivery of the mindfulness intervention. APJ oversaw all aspects of data collection. APZ, APJ, ED, $\mathrm{ABM}, \mathrm{NR}$, and $\mathrm{KC}$ contributed to different stages of data analyses. All authors wrote the manuscript and approved the manuscript for submission. 
Funding Information This research was supported by Department of Army grant \#W81XWH-12-2-0051 to APJ.

\section{Compliance with Ethical Standards}

Conflict of Interest APJ and SLR are co-developers and copyright holders of the Mindfulness-Based Attention Training (MBAT) program materials.

Ethics Statement The study was approved by the Institutional Review Board at the University of Miami with oversight from the Human Research Protections Office of the US Department of Defense. All participants provided informed consent.

Open Access This article is licensed under a Creative Commons Attribution 4.0 International License, which permits use, sharing, adaptation, distribution and reproduction in any medium or format, as long as you give appropriate credit to the original author(s) and the source, provide a link to the Creative Commons licence, and indicate if changes were made. The images or other third party material in this article are included in the article's Creative Commons licence, unless indicated otherwise in a credit line to the material. If material is not included in the article's Creative Commons licence and your intended use is not permitted by statutory regulation or exceeds the permitted use, you will need to obtain permission directly from the copyright holder. To view a copy of this licence, visit http://creativecommons.org/licenses/by/4.0/.

\section{References}

Adler, A. B., Litz, B. T., Castro, C. A., Suvak, M., Thomas, J. L., Burrell, L., McGurk, D., Wright, K. M., \& Bliese, P. D. (2008). A group randomized trial of critical incident stress debriefing provided to U.S. peacekeepers. Journal of Traumatic Stress, 21(3), 253-263. https://doi.org/10.1002/jts.20342.

Bijlsma, T., Muis, S., \& van Tilborg, A. (2019). Mindfulness in the Dutch military - train your brain. In Á. Rocha \& T. Guarda (Eds.), Developments and Advances in Defense and Security. MICRADS 2018. Smart innovation, systems and technologies (Vol. 94). Cham: Springer. https://doi.org/10.1007/978-94-6265-315-3_10.

Blacker, K., Hamilton, J., Roush, G., Pettijohn, K., \& Biggs, A. T. (2018). Cognitive training for military application: A review of the literature and practical guide. Journal of Cognitive Enhancement, 3(1), 3051. https://doi.org/10.1007/s41465-018-0076-1.

Bowen, D. J., Kreuter, M., Spring, B., Cofta-Woerpel, L., Linnan, L., Weiner, D., Bakken, S., Kaplan, C. P., Squiers, L., Fabrizio, C., \& Fernandez, M. (2009). How we design feasibility studies. American Journal of Preventive Medicine, 36(5), 452-457. https://doi.org/10. 1016/j.amepre.2009.02.002.

Crane, R., Brewer, J., Feldman, C., Kabat-Zinn, J., Santorelli, S., Williams, J., \& Kuyken, W. (2017). What defines mindfulnessbased programs? The warp and the weft. Psychological Medicine, 47(6), 990-999. https://doi.org/10.1017/S0033291716003317.

Deuster, P. A., \& Shoomaker, E. (2015). Mindfulness: A fundamental skill for performance sustainment and enhancement. Journal of Special Operations Medicine: a Peer Reviewed Journal for SOF Medical Professionals, 15(1), 93-99.

Doran, N., Hohman, M., \& Koutsenok, I. (2013). Motivational interviewing training in juvenile corrections: A comparison of outside experts and internal trainers. Legal and Criminological
Psychology, 18(2), 262-273. https://doi.org/10.1111/j.2044-8333. 2011.02036.x

Gamble, K. R., Vettel, J. M., Patton, D. J., Eddy, M. D., Davis, F. C., Garcia, J. O., Spangler, D. P., Thayer, J. F., \& Brooks, J. R. (2018). Different profiles of decision making and physiology under varying levels of stress in trained military personnel. International Journal of Psychophysiology, 131, 73-80. https://doi.org/10.1016/j. ijpsycho.2018.03.017.

Good, D. J., Lyddy, C. J., Glomb, T. M., Bono, J. E., Brown, K. W., Duffy, M. K., Baer, R. A., Brewer, J. A., \& Lazar, S. W. (2015). Contemplating mindfulness at work: An integrative review. Journal of Management, 42(1), 114-142. https://doi.org/10.1177/ 0149206315617003.

Gupta, S. K. (2011). Intention-to-treat concept: A review. Perspectives in Clinical Research, 2(3), 109-112. https://doi.org/10.4103/22293485.83221

Hofmann, W., Schmeichel, B. J., \& Baddeley, A. D. (2012). Executive functions and self-regulation. Trends in Cognitive Sciences, 16(3), 174-180. https://doi.org/10.1016/j.tics.2012.01.006.

Jha, A. P. (2002). Tracking the time-course of attentional involvement in spatial working memory: An event-related potential investigation. Cognitive Brain Research, 15(1), 61-69. https://doi.org/10.1016/ s0926-6410(02)00216-1.

Jha, A. P., Stanley, E. A., Kiyonaga, A., Wong, L., \& Gelfand, L. (2010). Examining the protective effects of mindfulness training on working memory capacity and affective experience. Emotion, 10(1), 54-64. https://doi.org/10.1037/A0018438.

Jha, A. P., Morrison, A. B., Dainer-Best, J., Parker, S., Rostrup, N., \& Stanley, E. (2015). Minds "At Attention": Mindfulness training curbs attentional lapses in military cohorts. PLoS One, 10(2), 119. https://doi.org/10.1371/journal.pone.0116889.

Jha, A. P., Morrison, A. B., Parker, S. C., \& Stanley, E. A. (2016). Practice is protective: Mindfulness training promotes cognitive resilience in high-stress cohorts. Mindfulness, 7(1), 1-13. https://doi.org/10. 1007/s12671-015-0465-9.

Jha, A. P., Witkin, J. E., Morrison, A. B., Rostrup, N., \& Stanley, E. (2017). Short-form mindfulness training protects against working memory degradation over high-demand intervals. Journal of Cognitive Enhancement, 1(2), 154-171. https://doi.org/10.1007/ s41465-017-0035-2.

Jha, A. P., Denkova, E., Zanesko, A. B., Witkin, J. E., Rooks, J., \& Roger, S. L. (2019). Does mindfulness training help working memory "work" better? Current Opinion in Psychology, 28, 273-278. https://doi.org/10.1016/j.copsyc.2019.02.012.

Johnson, D. C., Thom, N. J., Stanley, E. A., Haase, L., Simmons, A. N., Shih, P. A., Thompson, W. K., Potterat, E. G., Minor, T. R., \& Paulus, M. P. (2014). Modifying resilience mechanisms in at-risk individuals: A controlled study of mindfulness training in marines preparing for deployment. American Journal of Psychiatry, 171(8), 844-853. https://doi.org/10.1176/appi.ajp.2014.13040502.

Kabat-Zinn, J. (1990). Using the wisdom of your body and mind to face stress, pain, and illness. New York: Dell Publishing.

Lakens, D. (2013). Calculating and reporting effect sizes to facilitate cumulative science: A practical primer for $t$-tests and ANOVAs. Frontiers in Psychology, 26, 863. https://doi.org/10.3389/fpsyg. 2013.00863

Lieberman, H. R., Bathalon, G. P., Falco, C. M., Kramer, F. M., Morgan, C. A., \& Niro, P. (2005). Severe decrements in cognition function and mood induced by sleep loss, heat, dehydration, and undernutrition during simulated combat. Biological Psychiatry, 57(4), 422429. https://doi.org/10.1016/j.biopsych.2004.11.014.

Lutz, A., Jha, A. P., Dunne, J. D., \& Saron, C. (2015). Investigating the phenomenological matrix of mindfulness-related practices from a neurocognitive perspective. American Psychologist, 70(7), 632658. https://doi.org/10.1037/a0039585. 
Lyssenko, L., Muller, G., Kleindienst, N., Schmahl, C., Berger, M., Eifert, G., Kolle, A., Nesch, S., Ommer-Hohl, J., Weener, M., \& Bohus, M. (2016). Effectiveness of a mindfulness-based mental health promotion program provided by health coaches: A controlled multisite field trial. Psychotherapy and Psychosomatics, 85, 375-377. https://doi.org/10.1159/000447012.

Marx, P. (2019). Navigating dilemmas in training people to deliver noneight-week adapted mindfulness-based interventions. Mindfulness, 10(7), 1217-1221. https://doi.org/10.1007/s12671-019-01110-0.

Marx, B. P., Doron-LaMarca, S., Proctor, S. P., \& Vasterling, J. J. (2009). The influence of pre-deployment neurocognitive functioning on post-deployment PTSD symptom outcomes among Iraq-deployed Army soldiers. Journal of the International Neuropsychological Society, 15(6), 840-852. https://doi.org/10.1017/ S1355617709990488.

Meland, A., Ishimatsu, K., Pensgaard, A. M., Fonne, V., Garde, A. H., \& Harris, A. (2015). Impact of mindfulness training on physiological measures of stress and objective measures of attention control in a military helicopter unit. International Journal of Aviation Psychology, 25(3-4), 191-208. https://doi.org/10.1080/10508414. 2015.1162639.

Morey, R. A., Petty, C. M., Cooper, D. A., Labar, K. S., \& McCarthy, G. (2008). Neural systems for executive and emotional processing are modulated by symptoms of posttraumatic stress disorder in Iraq War veterans. Psychiatry Research, 162(1), 59-72. https://doi.org/10. 1016/j.pscychresns.2007.07.007.

Morgan 3rd, C. A., Doran, A., Steffian, G., Hazlett, G., \& Southwick, S. M. (2006). Stress-induced deficits in working memory and visuoconstructive abilities in Special Operations soldiers. Biological Psychiatry, 60(7), 722-729. https://doi.org/10.1016/j. biopsych.2006.04.021.

Morrison, A. B., Goolsarran, M., Rogers, S. L., \& Jha, A. P. (2014). Taming a wandering attention: Short-form mindfulness training in student cohorts. Frontiers in Human Neuroscience, 7(897), 1-12. https://doi.org/10.3389/fnhum.2013.00897.

Mrazek, M. D., Franklin, M. S., Phillips, D. T., Baird, B., \& Schooler, J. W. (2013). Mindfulness training improves working memory capacity and GRE performance while reducing mind wandering. Psychological Science, 24(5), 776-781. https://doi.org/10.1177/ 0956797612459659.

Robertson, I. H., Manly, T., Andrade, J., Baddeley, B. T., \& Yiend, J. (1997). 'Oops!': Performance correlates of everyday attentional failures in traumatic brain injured and normal subjects. Neuropsychologia, 35(6), 747-758. https://doi.org/10.1016/s00283932(97)00015-8.

Schulz, K. F., Altman, D. G., \& Moher, D. (2010). CONSORT 2010 statement: Updated guidelines for reporting parallel group randomised trials. BMC Medicine, 8, 18. https://doi.org/10.1186/ 1741-7015-8-18.

Smallwood, J., \& Schooler, J. W. (2015). The science of mind wandering: Empirically navigating the stream of consciousness. Annual Review of Psychology, 66, 487-518. https://doi.org/10.1146/annurev-psych010814-015331.

Stanislaw, H., \& Todorov, N. (1999). Calculation of signal detection theory measures. Behavior Research Methods, Instruments, \& Computers, 31(1), 137-149. https://doi.org/10.3758/BF03207704.

Stanley, E. A. (2014). Cultivating the mind of a warrior. Inquiring mind. Retrieved from http://www.inquiringmind.com/Articles/ CultivatingMindOfWarrior.html

Stanley, E. A., \& Jha, A. P. (2009). Mind fitness and mental armor: Enhancing performance and building warrior resilience. Joint Force Quarterly, 55, 144-151.

Stanley, E. A., Schaldach, J. M., Kiyonaga, A., \& Jha, A. P. (2011). Mindfulness-based mind fitness training: A case study of a highstress predeployment military cohort. Cognitive and Behavioral Practice, 18(4), 566-576. https://doi.org/10.1016/j.cbpra.2010.08. 002.

U.S. Army. (2014). Comprehensive soldier and family fitness. Army regulation 350-53. Washington, DC: Headquarters, Department of the Army. Retrieved from www.army.mil/e2/downloads/rv7/r2/ policydocs/r350_53.pdf

Vasterling, J. J., Brailey, K., Constans, J. I., \& Sutker, P. B. (1998). Attention and memory dysfunction in posttraumatic stress disorder. Neuropsychology, 12(1), 125-133. https://doi.org/10.1037/08944105.12.1.125.

Wilson, K. M., Head, J., de Joux, N. R., Finkbeiner, K. M., \& Helton, W. S. (2015). Friendly fire and the sustained attention to response task. Human Factors, 57(7), 1219-1234. https://doi.org/10.1177/ 0018720815605703.

Zanesco, A. P., King, B. G., MacLean, K. A., \& Saron, C. D. (2013). Executive control and felt concentrative engagement following intensive meditation training. Frontiers in Human Neuroscience, 7, 566. https://doi.org/10.3389/fnhum.2013.00566.

Zanesco, A. P., King, B. G., MacLean, K. A., \& Saron, C. D. (2018). Cognitive aging and long-term maintenance of attentional improvements following meditation training. Journal of Cognitive Enhancement, 2(3), 259-275. https://doi.org/10.1007/s41465-0180068-1.

Zanesco, A. P., Denkova, E., Rogers, S. L., MacNulty, W. K., \& Jha, A. P. (2019). Mindfulness training as cognitive training in high-demand cohorts: An initial study in elite military servicemembers. Progress in Brain Research, 244, 323-354. https://doi.org/10.1016/bs.pbr. 2018.10.001.

Zhang, N. A., Rudi, J. H., \& Gerwirtz, A. H. (2018). Parent engagement in online mindfulness exercises within a parent training program for post-deployed military families. Mindfulness, 9(3), 725-736. https:// doi.org/10.1007/s12671-017-0810-2.

Publisher's Note Springer Nature remains neutral with regard to jurisdictional claims in published maps and institutional affiliations. 